\title{
Köhler theory for a polydisperse droplet population in the presence of a soluble trace gas, and an application to stratospheric STS droplet growth
}

\author{
H. Kokkola, S. Romakkaniemi, and A. Laaksonen \\ Department of Applied Physics, University of Kuopio, Finland \\ Received: 23 April 2003 - Published in Atmos. Chem. Phys. Discuss.: 26 June 2003 \\ Revised: 25 September 2003 - Accepted: 12 November 2003 - Published: 3 December 2003
}

\begin{abstract}
We consider the equilibrium behavior of a polydisperse aqueous droplet population as a function of relative humidity $(\mathrm{RH})$ when a soluble trace gas, such as nitric acid, is present in the system. The droplet population experiences a splitting when the RH is increased sufficiently. This splitting is not related to the traditional Köhler activation of cloud droplets, as it may occur at relative humidities below $100 \%$. Remarkably, the splitting always takes place in such a way that the largest size class of the (discretized) droplet population starts taking up the soluble trace gas efficiently, growing steeply as a function of $\mathrm{RH}$, and forcing the smaller droplets to shrink. We consider this behavior in terms of open and closed system Köhler curves (open system referring to one in which the trace gas concentration remains constant and closed system to one in which the gas concentration decreases as a result of uptake of the trace gas). We show how the open and closed system Köhler curves are related, and that the splitting of the population can be explained in terms of closed system curves crossing the Köhler maxima of the open system curves. We then go on to consider time-dependent situations, and show that due to gas-phase mass transfer limitations, the splitting of the size distributions moves toward smaller sizes as the rate of RH increase becomes more rapid. Finally, we consider stratospheric supercooled ternary solution droplet populations, and show that the splitting described using the new theory may lead to formation of bimodal size distributions in the stratosphere.
\end{abstract}

\section{Introduction}

For decades the formation of cloud droplets has been described using Köhler theory (Köhler, 1936; Seinfeld and Pandis, 1998). The theory assumes that the droplets consist

Correspondence to: $\mathrm{H}$. Kokkola

(harri.kokkola@uku.fi) of a binary mixture of completely dissociated electrolytic salt and water. According to the theory, the droplets are in the thermodynamical equilibrium until the saturation ratio of the water vapour reaches a critical value, after which the droplets are "activated" and they grow spontaneously to cloud droplets.

In recent studies it has been shown that there are chemical factors that can affect the equilibrium saturation ratio of the particles and have effect on the number of particles that are activated to cloud droplets. Soluble hygroscopic gases, slightly soluble solutes, and surface tension depression by organic substances may influence cloud droplet activation (Kulmala et al., 1993; Shulman et al., 1996; Laaksonen et al., 1998). Also irreversible chemical reactions, mainly sulfate production, can contribute to cloud droplet formation (Kokkola et al., 2003a). In this article we examine the effects of soluble hygroscopic gases on the growth of droplet populations. We first extend the theoretical considerations of Laaksonen et al. (1998) to describe the equilibrium behavior of a polydisperse droplet distribution as a function of relative humidity in the presence of a soluble trace gas, and note that the population may split into two parts although no cloud activation in the traditional sense takes place. We then examine, using a cloud model, how this behavior is modified due to gas-phase kinetics. Finally, we show that ternary stratospheric droplet populations may experience the splitting and become bimodal at low cooling rates.

\section{Numerical models}

For the calculations we used two types of models, an equilibrium model and a cloud model (non-equilibrium model). The equilibrium model calculates the thermodynamical equilibrium at given temperature and relative humidity for a lognormal aerosol particle population. The equilibrium is calculated among solids, liquids, ions, and gases over multiple 
discrete size bins. The model includes the aerosol inorganics model AIM (Clegg et al., 1998) for calculation of activity coefficients as functions of solution concentrations and temperature.

The cloud model is a moving sectional box model for simulating the evolution of physical and chemical properties of an aerosol population (Kokkola et al., 2003b). It has been developed to simulate cloud or fog droplet formation. The physicochemical processes affecting the aerosol population and included in the model are condensation and evaporation of water vapor and trace gases, gas emissions, and reversible and irreversible chemical reactions producing sulfate in the liquid phase. The model also includes modules for equilibrating the aerosol population with water vapor and trace gases, and for calculating the optical properties of the aerosol population. In the cloud model, activity coefficients are calculated either with the modified version of the thermodynamical equilibrium model EQUISOLV II (Jacobson, 1999), or with AIM.

The model was slightly modified when the calculations were made in stratospheric conditions $(<200 \mathrm{~K})$. In these conditions, the equilibrium model AIM was used. In stratospheric conditions the droplets are highly concentrated, so the effect of nitric acid and sulfuric acid was taken into account when calculating the surface tension and the density of the droplets. For this, we used parameterizations developed by Martin et al. (2000) for surface tension and density of $\mathrm{H}_{2} \mathrm{O} / \mathrm{HNO}_{3} / \mathrm{H}_{2} \mathrm{SO}_{4}$ droplets. Also, the diffusion coefficient was calculated using an equation valid at low concentrations, given by Rizi and Visconti (1999).

\section{Equilibrium growth of monodisperse aerosol}

When soluble gases such as nitric acid $\left(\mathrm{HNO}_{3}\right)$ or hydrochloric acid $(\mathrm{HCl})$ are dissolved in aqueous salt solution droplets, the equilibrium saturation ratio of the droplets is lowered. The Köhler curve maximum is subsequently depressed, and if the concentration of the soluble gas is high, the maximum can occur at less than $100 \%$ relative humidity (Kulmala et al., 1993). The equations describing the equilibria of a solution droplet with water vapor and a soluble gas are:

$$
\begin{aligned}
& S_{w}=x_{w} f_{w} \exp \left(2 \sigma v_{w} / R T r\right) \\
& S_{a}=x_{a} f_{a} \exp \left(2 \sigma v_{a} / R T r\right) .
\end{aligned}
$$

Here the subscripts $w$ and $a$ denote water and the soluble gas in question, respectively, $S$ is saturation ratio, $x$ is mole fraction, $f$ is the rational activity coefficient, $r$ is the droplet radius, $\sigma$ denotes surface tension, $v$ is molar volume, $R$ is the gas constant, and $T$ is temperature.

In Laaksonen et al. (1998) we derived, starting from Eqs. (1)-(2), equations giving the equilibrium radius of the droplet at given saturation ratio of water, in two special cases. The first case is an open system with respect to the soluble gas, i.e. one in which the gas phase concentration of species $a$ stays constant:

$S_{w} \simeq 1+\frac{a_{w}}{r}-\frac{b_{s}}{r^{3}}-v_{a}\left(P_{a} K_{a}\right)^{1 / 2}$.

Here $a_{w}=2 \sigma v_{w} / R T$, and $b_{s}=3 M_{w} v_{s} n_{s} / 4 \pi \rho_{w}$ with $M_{w}$ and $\rho_{w}$ the molar mass and density of water, $v_{s}$ and $v_{a}$ the number of ions the salt and the soluble gas dissociate into, and $P_{a}$ and $K_{a}$ the partial pressure, and Henry's law coefficient of the soluble gas, respectively. Thus, at high relative humidities, the traditional Köhler curve of a salt solution droplet is depressed by a constant amount, determined by the last term on the right hand side of the equation, when a soluble species is present at constant gas-phase concentration.

The second case considered by Laaksonen et al. (1998) was a closed system, in which the total amount of the soluble species (gas phase + dissolved) is assumed constant. The resulting equilibrium equation is then

$S_{w} \simeq 1+\frac{a_{w}}{r}-\frac{b_{s}}{r^{3}}-\frac{2 n_{t}}{b_{a} r^{3}\left(1+\left(1+\frac{4 n_{t}}{C \beta r^{6}}\right)^{1 / 2}\right)}$

where $C$ is aerosol concentration $n_{t}$ is the total number of moles of the soluble substance per unit volume, $b_{a}=4 \pi \rho_{w} / 3 M_{w} v_{a}$ and $\beta=K_{a} R T\left(\rho_{w} 4 \pi / 3 M_{w}\right)^{2}$. Below, we shall call curves produced by Eqs. (3) and (4) open and closed system Köhler curves, respectively. (Note that Eqs. (3) and (4) were derived subject to a number of approximations, and they are accurate at high relative humidities only. At RH $<99 \%$, the equilibria should be solved numerically from Eqs. (1) and (2), and the appropriate condition referring to open or closed system.)

The grey lines in Fig. 1 show the open system Köhler curves for a $75 \mathrm{~nm}$ ammonium sulfate particle when a varying amount between 0 and $10 \mathrm{ppb}$ of nitric acid is in the gas phase at $298 \mathrm{~K}$ temperature and $1 \mathrm{~atm}$ pressure. Thus, in an open system with $10 \mathrm{ppb}$ of nitric acid in the gas phase, the droplets would be activated at slightly less than $99.5 \%$ relative humidity.

In the closed system, the Köhler curve approaches that predicted by the traditional Köhler theory as the RH grows, the equilibrium size increases, and nitric acid is depleted from the gas phase (see Laaksonen et al., 1998). The black solid line in Fig. 1 shows the modified Köhler curve in a closed system for the $75 \mathrm{~nm}$ ammonium sulfate particles with a number concentration of $100 \mathrm{~cm}^{-3}$. In the calculation of this curve, the initial gas-phase nitric acid mixing ratio was $10 \mathrm{ppb}$. As $\mathrm{RH}$ increases and $\mathrm{HNO}_{3}$ is depleted from the gas-phase, the closed system Köhler curve crosses a succession of open system curves corresponding to a smaller and smaller concentration of gas-phase nitric acid. For droplets larger than about $1 \mu \mathrm{m}$ the crossing points reside on the unstable (decreasing) side of the open system curves, however, the resulting closed system Köhler curve represents stable equilibria. Thus, the droplet can be over micron sized but still 


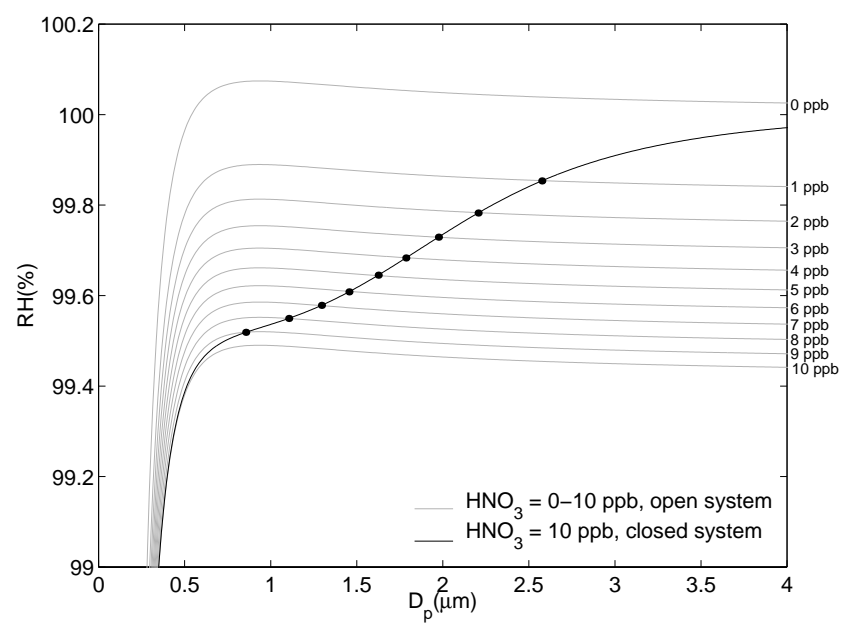

Fig. 1. Modified Köhler curves for an ammonium sulfate particle with dry diameter of $75 \mathrm{~nm}$ in an open system when the gas phase concentrations is varied between 0 and $10 \mathrm{ppb}$ and the modified Köhler curve when $10 \mathrm{ppb}$ of nitric acid is in the gas phase in a closed system.

be unactivated at relative humidities below 100\% (Kulmala et al., 1997; Laaksonen et al., 1998). Note that Konopka and Vogelsberger (1997) have studied, by considering Helmholtz free energies, the relation between systems that are open and closed with respect to condensable water.

\section{Equilibrium growth of a polydisperse aerosol popula- tion}

In this section we consider the equilibrium behavior of polydisperse aerosols as a function of $\mathrm{RH}$ when nitric acid is present in the system. From now on, the approximations of Eqs. (3) and (4) will be omitted, and the equilibria are solved from Eqs. (1) and (2), using appropriate thermodynamic models to obtain the activity coefficients.

Figure 2 shows the effect of $\mathrm{HNO}_{3}$ on the equilibrium saturation ratios of a polydisperse ammonium sulfate-water droplet population. The dashed lines are the traditional Köhler curves for different sized ammonium sulfate particles. The solid lines are the modified open system Köhler curves for the same particles when $0.1 \mathrm{ppb}$ of $\mathrm{HNO}_{3}$ is in the gas-phase. The figure shows that with sufficiently large particles, the maxima of the open system Köhler curves are below unity. Thus, in an open system with $0.1 \mathrm{ppb}$ nitric acid, the largest droplets of Fig. 2 would be activated at about $99.95 \%$ relative humidity.

In a closed system with a polydisperse aerosol population, the partitioning of $\mathrm{HNO}_{3}$ to a single size class affects the equilibria of all other size classes, i.e. the closed system Köhler curves for the different droplet sizes are not independent of each other. Therefore, closed system Köhler curves

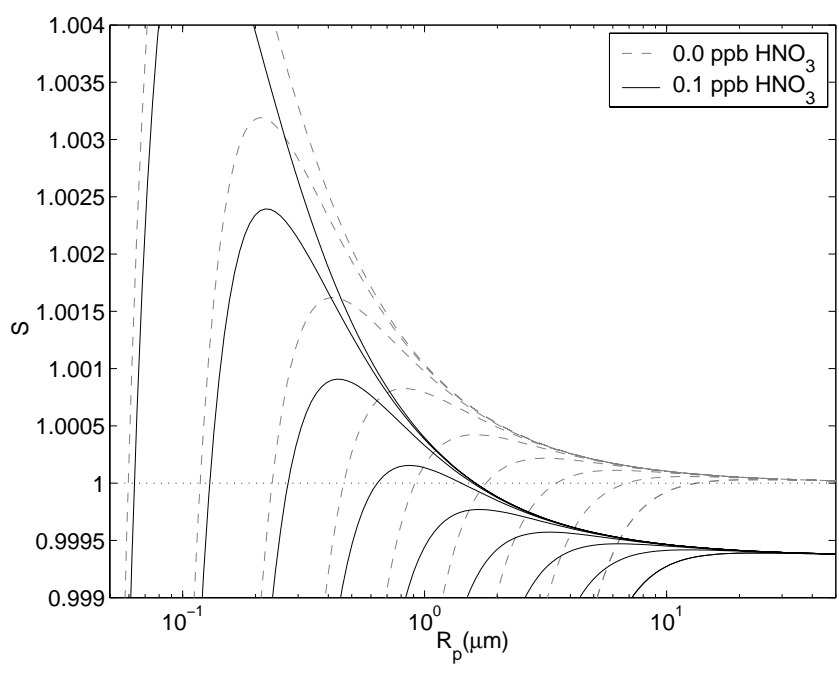

Fig. 2. Equilibrium saturation ratio for a case without $\mathrm{HNO}_{3}$ and with $0.1 \mathrm{ppb}$ of $\mathrm{HNO}_{3}$ in the gas phase (open system).

have to be calculated iteratively for a polydisperse droplet population. One way of carrying out the iteration is to take the gas-phase $\mathrm{HNO}_{3}$ concentration to be the iterated variable. Thus, at given RH and with a given total amount (gas-phase + aqueous phase) of $\mathrm{HNO}_{3}$ per unit volume of air, a guess is made for the remaining gas phase acid concentration, and equilibria are calculated for all droplet sizes as if the system was open. If the sum of the gas and aqueous phase acids does not match the correct total amount of $\mathrm{HNO}_{3}$, a new gas phase concentration is selected, and the iteration continues until the sum matches the correct value.

As an example, we calculated the behavior of the equilibrium size distribution of an ammonium sulfate particle population as a function of relative humidity in a closed system. The geometrical mean diameter for the size distribution was $10 \mathrm{~nm}$, the total number concentration of the particles was $500 \mathrm{~cm}^{-3}$ and the geometric standard deviation was 2.0 . In the gas phase, there was initially $5 \mathrm{ppb}$ of $\mathrm{HNO}_{3}$. The initial relative humidity was $95 \%$ at $278.15 \mathrm{~K}$.

Figure $3 \mathrm{a}$ shows the equilibrium radius for each size bin at the relative humidity range $95 \%-100 \%$. Above the $\mathrm{RH}$ of $98,5 \%$, the size distribution splits as the radius of the largest size class starts increasing very steeply as a function of $\mathrm{RH}$, whereas the radii of the smaller size classes actually decrease. The splitting can be understood by considering the open system Köhler curves. Figure $3 b$ is magnification from Fig. 3a and shows the equilibrium radii of the two largest size bins (solid lines) for the relative humidity range $98.55 \%$ $98.8 \%$. The grey lines in the figure are the open system Köhler curves corresponding to the gas phase concentration of $\mathrm{HNO}_{3}$, calculated for each value of relative humidity in the closed system (black dots on the solid lines). 


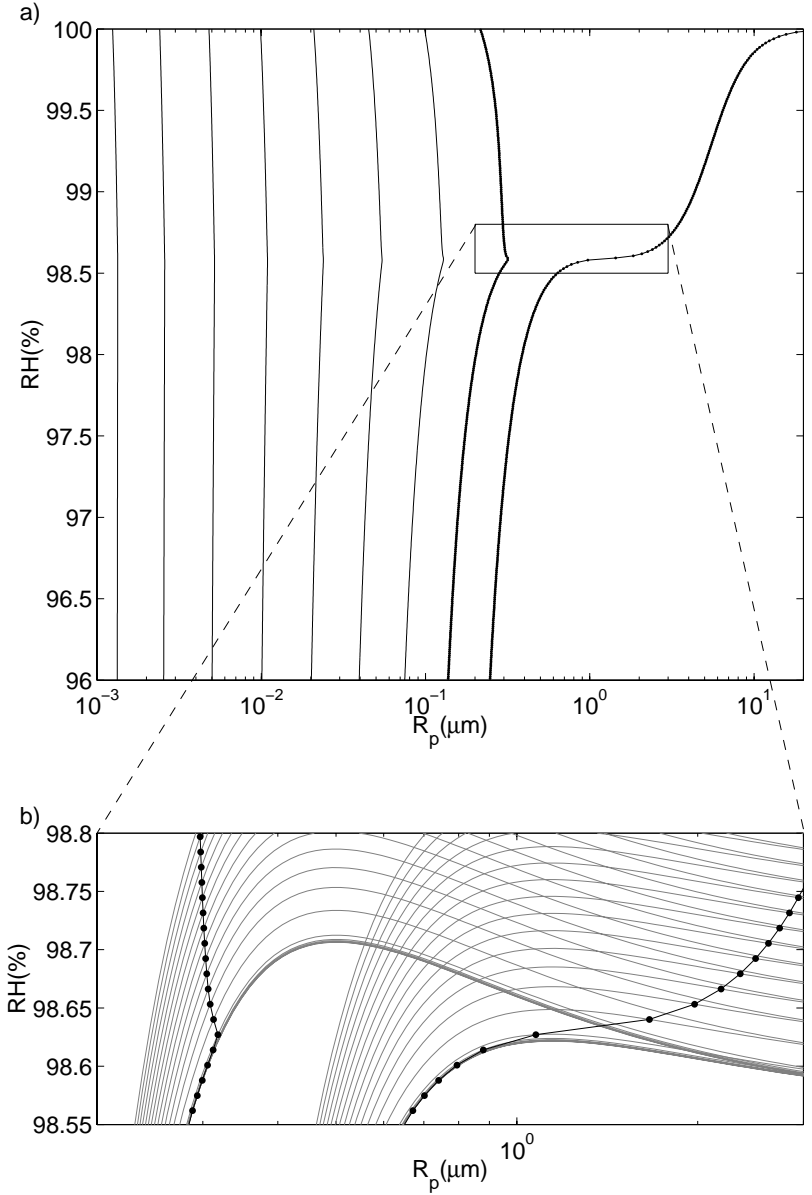

Fig. 3. Equilibrium size distribution calculated as a function of relative humidity (RH). The second figure shows the equilibrium size distribution (lines with dots) with the corresponding open system Köhler curves.

Below about $98.6 \%$ relative humidity the open system Köhler curves are very close to each other. This is because the $\mathrm{HNO}_{3}$ is partitioned mostly in the gas phase and its gas phase concentration decreases very little as a function of $\mathrm{RH}$. However, when the relative humidity increases to over $98.6 \%$, the largest bin approaches a maximum at its open Köhler curves. Around the maximum and to the right from it the slopes of the open Köhler curves become very gentle, resulting in greatly increased uptake of water and $\mathrm{HNO}_{3}$ for a small increase in RH. This, in turn, forces the smaller droplets to evaporate some of their water and nitric acid, and their sizes begin to decrease. Furthermore, the droplets in the smaller size bins will never exceed their open Köhler curve maxima. Thus, at the relative humidity where the largest size bin exceeds the open system Köhler maximum, a gap forms between the largest size bin and the smaller size bins. At higher relative humidities there is no such solution for the equilibrium, where the largest size bin would be on the ascending part of an open system equilibrium curve.

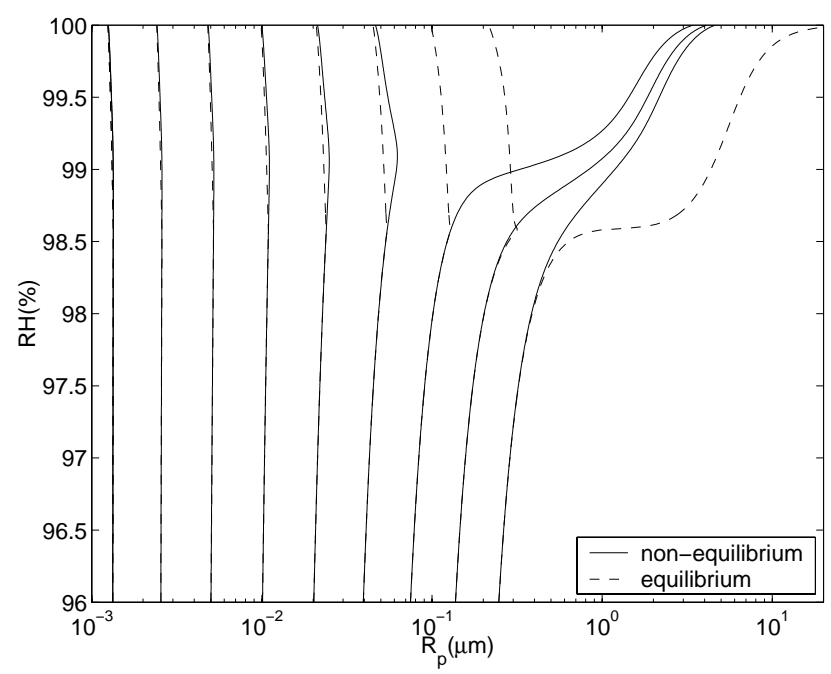

Fig. 4. Size distribution calculated as a function of relative humidity when diffusion is taken into account (solid lines) and the equilibrium size distribution (dashed lines)

The physical reason for the splitting described above is undoubtedly that the system minimizes its free energy in analogy with the Ostwald ripening effect, whereby larger crystals grow in the expense of smaller ones in supersaturated liquid solutions.

\section{Non-equilibrium growth}

In real, time-dependent situations, the equilibrium growth theory formulated above can be considered as a limit that is approached asymptotically when the growth rate of relative humidity becomes slower and slower. In the atmosphere, the changes of RH are so rapid that when soluble trace gases are condensing into aqueous droplets, the droplets are practically always out of equilibrium during the growth. (To be more precise, they may retain near equilibrium with water vapor but not with the trace gases). This is a simple consequence of mass transfer limitations with species having mixing ratios on the order of a few ppb.

If the limitations of mass transfer are taken into account when considering the splitting of the size distribution (Fig. 3), it is obvious that the growth of the largest size class from a few hundred $\mathrm{nm}$ to about one micrometer will in many cases take more time than it takes for the RH to increase above the open system Köhler maxima of some of the smaller size classes. In practice, then, the splitting of the size distribution may occur at smaller and smaller sizes the more rapidly the relative humidity is increasing (or temperature decreasing).

To investigate the non-equilibrium effects on the splitting of the size distribution, we made a cloud model simulation 


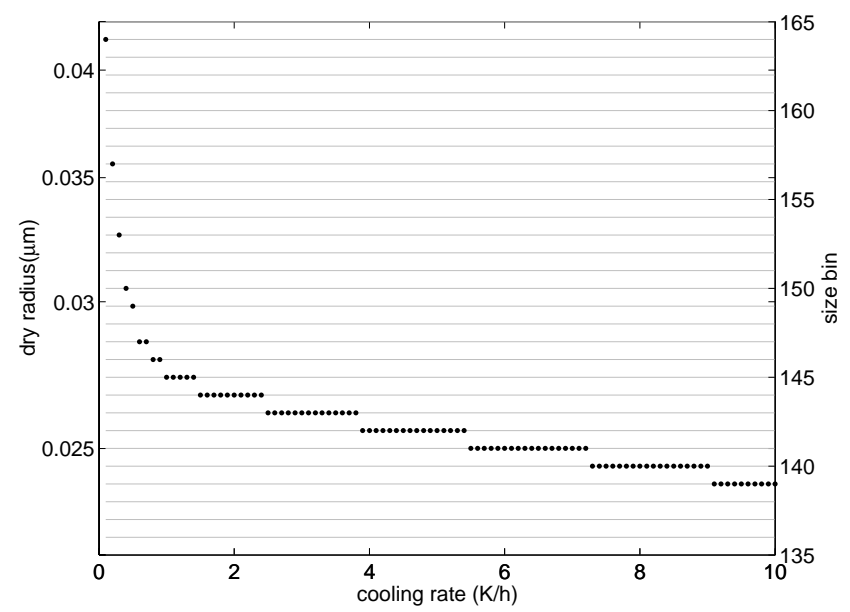

Fig. 5. The size bin under which a gap in the size distribution appears calculated and the corresponding dry radius of the particles in the size bin as a function of cooling rate.

with the same parameters that were used to calculate the equilibrium size distribution shown in Fig. 3a. The rate of temperature decrease was chosen to be $1 \mathrm{~K} / \mathrm{h}$. Figure 4 shows the evolution of the size distribution as a function of relative humidity and is represented by solid lines. For comparison, the equilibrium size distribution is also included in the figure (dashed lines). Figure 4 shows that when the gas-phase diffusion of the condensing species is accounted for, two more size bins grow to over micron sized droplets compared to the equilibrium growth calculation. The relative humidity is still below $100 \%$, so the particles are not activated in the traditional sense although a gap has appeared in the size distribution.

To investigate the effect of the cooling rate, we made calculations for the same size distribution of ammonium sulfate particles using different cooling rates in the range 0.1$10 \mathrm{~K} / \mathrm{h}$. The size distribution consisted of 200 size bins. The model was initialized at the temperature of $278.15 \mathrm{~K}$ and 95\% relative humidity. The temperature was then decreased linearly until the relative humidity reached $99 \%$ at approximately $277.6 \mathrm{~K}$. In all the simulations, the initial gas phase volume mixing ratio of $\mathrm{HNO}_{3}$ was $5 \mathrm{ppb}$. At the end of the simulation, we determined the location in the size distribution where the gap appears.

Figure 5 shows the index of the size bin below which the gap in the size distribution occurs. The size bin is shown as a function of cooling rate and the index for each cooling rate is denoted by a dot. The dry radii corresponding to the size bins are denoted by grey solid lines.

At extremely slow cooling rates, the location of the gap appears to be very much dependent on the cooling rate. When the cooling rate is increased, the location of the gap varies very little since the growth of the smaller droplets is more
Table 1. Parameters for different simulations.

\begin{tabular}{cll}
\hline Case & cooling rate (K/day) & number concentration $\left(\mathrm{cm}^{-3}\right)$ \\
\hline A & 0.3 & 10 \\
B & 0.3 & 20 \\
C & 1.0 & 10 \\
D & 1.0 & 20 \\
\hline
\end{tabular}

rapid, and, on the other hand, the difference in the heights of the open system Köhler curve maxima is bigger for smaller size bins (see Fig. 3).

Note, that with tropospheric clouds the RH reaches its maximum value too rapidly for the splitting of the population caused by $\mathrm{HNO}_{3}$ to have much bearing on the cloud drop activation. Rather, the nitric acid effect described e.g. by Kulmala et al. (1993) is caused by the fact that small drops, with higher surface-to-volume ratio, are more effective in collecting $\mathrm{HNO}_{3}$ molecules from the gas. The amount of water soluble material is therefore increased relatively more in small drops than in large drops by the time the peak value of RH is reached, leading to enhanced activation and increased cloud drop number concentration.

\section{Selective growth in stratospheric clouds}

The effect of the semi-volatile species on the equilibrium size of the droplets at given relative humidity is enhanced at low temperatures. In the stratosphere, nitric acid mixing ratios can be fairly high, and at temperatures below $200 \mathrm{~K}$ the uptake of $\mathrm{HNO}_{3}$ is significant already at low relative humidities (Tabazadeh et al., 1994; Kärcher and Solomon, 1999). In such conditions, the open system Köhler maximum of the largest droplets is lowered to about 30\% RH. In principle, the largest droplets could then grow to micrometer size range when that relative humidity is reached.

We made simulations for typical stratospheric conditions using a droplet population initially consisting of dry sulfuric acid, with geometrical mean diameter of $0.08 \mu \mathrm{m}$ and geometrical standard deviation of 1.7, and applying two different number concentrations, 10 and $20 \mathrm{~cm}^{-3}$. The number concentrations for different simulations are given in Table 1. The initial concentrations of water and nitric acid in the particle phase were calculated by equilibration. The gas phase volume mixing ratio for water was $4.5 \mathrm{ppm}$ and for $\mathrm{HNO}_{3}$ it was $12 \mathrm{ppb}$.

In the beginning of the simulation, the initial temperature was $193.15 \mathrm{~K}$, relative humidity was $24 \%$ and pressure was 60 mbar. The temperature was decreased linearly using two different cooling rates, $0.3 \mathrm{~K} /$ day and $1.0 \mathrm{~K} /$ day until relative humidity reached $45 \%$, at approximately $189.15 \mathrm{~K}$. These rates correspond to seasonal cooling which, according to Tabazadeh et al. (2000), can persist for days or weeks 


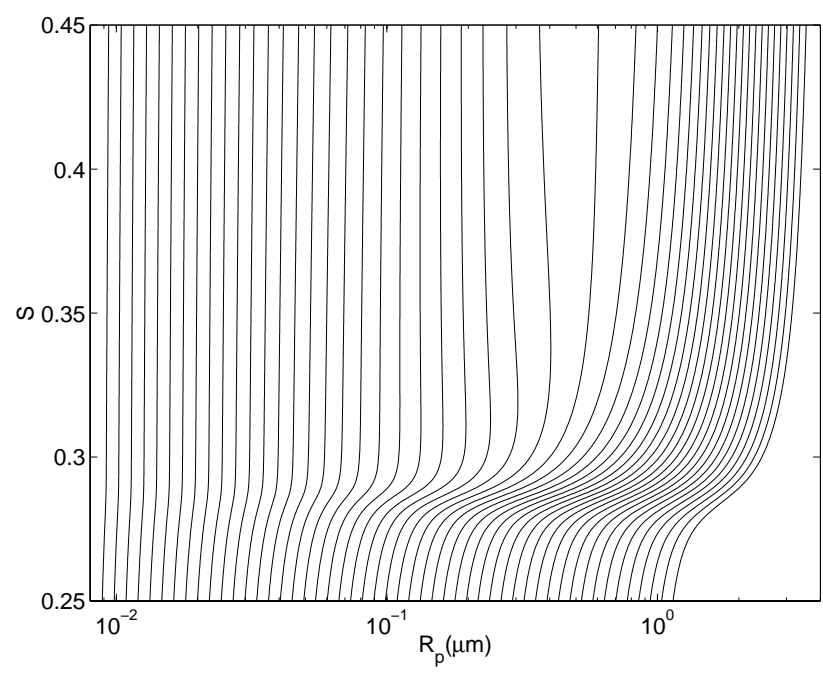

Fig. 6. The evolution of a size distribution in stratospheric conditions as a function of saturation ratio $S$.

even in the Arctic stratosphere. At some point, continuous cooling will lead to formation of large particles which will sediment, experiencing changing ambient conditions. However, at 68 mbar, effective sedimentation takes place only when the particle radii reach sizes of 6-8 $\mu \mathrm{m}$ (Tabazadeh et al., 2000). In our simulations - intended as a qualitative analysis of the condensation mechanisms that may occur the radii of the droplets remain below $2 \mu \mathrm{m}$, and thus it should be sufficient to describe the system as closed. The cooling rates for the different simulations are given in Table 1 .

Figure 6 shows the evolution of the size distribution for simulation case $A$ as a function of water vapour saturation ratio. In this simulation, we can clearly see the selective growth as the relative humidity increases. At slightly below $30 \%$ relative humidity, nitric acid starts to condense in the droplets and the size distribution gets wider. In that region, the largest particles approach the open system Köhler curve maxima. After relative humidity is further increased, the gap appears between the radii of $0.4-0.6 \mu \mathrm{m}$, indicating that the largest droplets have exceed the open system Köhler maxima and grow rapidly, simultaneously forcing the smaller droplets to evaporate some of their water and nitric acid.

The selective growth of the particle population changes the shape of the size distribution. Figure 7 shows how the initially unimodal distribution has developed into a bimodal distribution at $45 \%$ relative humidity. Figure 8 shows the final size distributions at $35 \%$ relative humidity for all four cases A-D.

From Fig. 8 we can see that for the equal cooling rates, the gap in the size distribution appears at the same size range independent of the number concentration. For the higher cooling rates (cases $\mathrm{C}$ and D) smaller droplets reach their open system Köhler maxima and so for these cases the number distribution in the larger mode is higher.

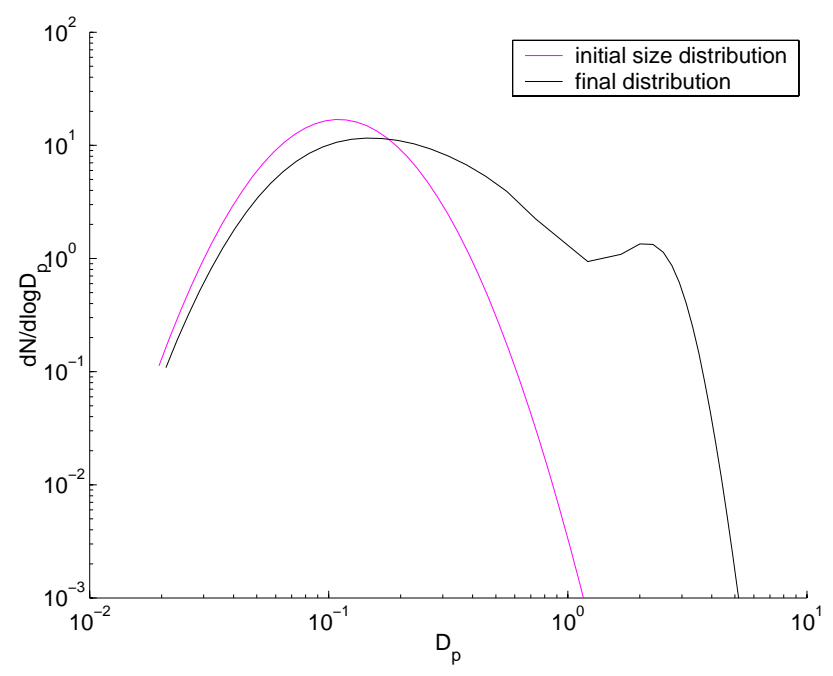

Fig. 7. The initial number size distribution (magenta line) and the final size distribution (black line) at $45 \%$ relative humidity.

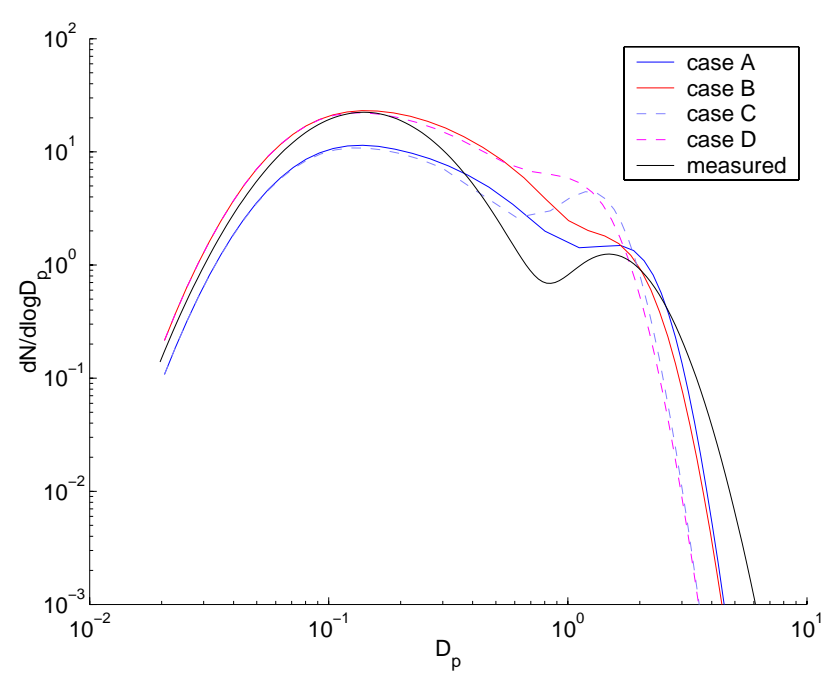

Fig. 8. The number size distribution at $35 \%$ relative humidity for cases A-D and the measured size distribution by Voigt et al. (2000).

For the cases with lower number concentration, the gap is more distinct. This is because the nitric acid concentration is higher in the droplets and thus the growth of the droplets in the larger mode is enhanced.

The calculated molar ratio of $\mathrm{H}_{2} \mathrm{O}: \mathrm{HNO}_{3}$ in the liquid phase for all the cases reaches a minimum at about $30 \%$ relative humidity after which the droplets are diluted with respect to nitric acid. Figure 9 shows the molar ratio $\mathrm{H}_{2} \mathrm{O}: \mathrm{HNO}_{3}$ at $30 \% \mathrm{RH}$ for all the size bins. For case $\mathrm{A}$, the minimum value is 3.58 and for all the cases the minimum is less than 3.7. The molar ratio increases toward the end of the simulation, but still at 35\% RH, the minimum molar ratio in the droplets is under 4 for all the cases. 


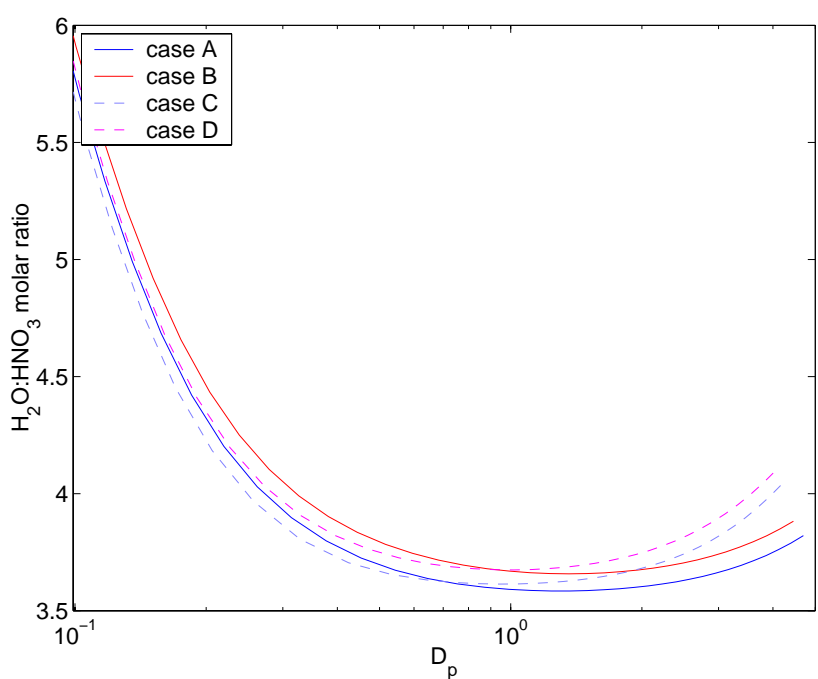

Fig. 9. Liquid phase molar ratio of $\mathrm{H}_{2} \mathrm{O}: \mathrm{HNO}_{3}$ at $30 \%$ relative humidity.

Measurements have shown bimodal size distributions to exist in the polar stratosphere. The black line in Fig. 8 shows a size distribution fitted to measured distribution for which the large particles were interpreted to consist of nitric acid trihydrate (NAT) (Voigt et al., 2000). The mean diameters for the modes in the fitted size distribution are $0.13 \mu \mathrm{m}$ and $1.52 \mu \mathrm{m}$, the geometrical standard deviations are 1.50 and 1.45 and the number concentrations are $16 \mathrm{~cm}^{-3}$ and $0.5 \mathrm{~cm}^{-3}$.

Interestingly, a comparison of the final distributions of cases A and B to the measured NAT size distribution shows that the mean diameters of the two modes match fairly well. For the model case B, also the number concentrations in the two modes match well with the measured data. This comparison shows that bimodality of the size distribution and measured $\mathrm{H}_{2} \mathrm{O}: \mathrm{HNO}_{3}$ molar ratios between 3 and 3.5 are not necessarily sufficient to prove that the larger mode is composed of NAT. With the cloud observed by Voigt et al. (2000), lidar depolarization measurements indicated that the particles were solid. However, the question remains whether the bimodality of the distribution was caused by the freezing itself which took place in a lee wave event two hours before the observations. The alternative explanation, suggested by our calculations, would be that the splitting of the distribution took place during slow cooling before the lee wave, and that the freezing to NAT occurred in the larger droplet mode which had a favorable $\mathrm{H}_{2} \mathrm{O}: \mathrm{HNO}_{3}$ molar ratio between 3.5 and 4.

In our simulations, the splitting of the distribution took place after 5 days; without knowledge of the airmass history, we cannot assess whether such a cooling period actually took place. In any case, regardless of what may have caused the

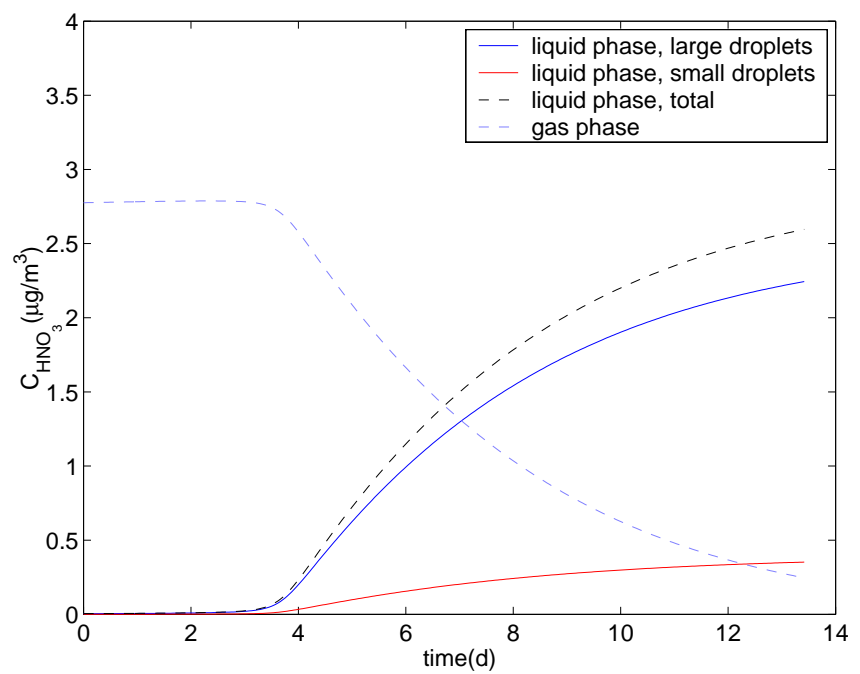

Fig. 10. Liquid phase concentration of $\mathrm{HNO}_{3}$ in large droplets (dashed line), small droplets (dash-dot line), total liquid phase concentration of $\mathrm{HNO}_{3}$ and the gas phase concentration of $\mathrm{HNO}_{3}$ as a function of time.

splitting of the distribution observed by Voigt et al. (2000), it seems clear to us that the mechanism presented in this paper is operational in the stratosphere and may under suitable conditions produce bimodal droplet distributions and possibly affect subsequent NAT cloud formation.

\section{Removal of gaseous nitric acid by liquid droplets in the stratosphere}

Carslaw et al. (1997) have shown by thermodynamical equilibrium calculations, that the Kelvin effect will affect the partitioning of nitric acid in stratospheric droplets so that its concentration is high in droplets with size $\sim 1 \mu \mathrm{m}$ in radius and and low in small droplets with size $\sim 0.01 \mu \mathrm{m}$. The kind of selective growth shown here will further increase the concentration difference between the large and the small droplets. Also, in contrast to equilibrium model results, as Fig. 9 illustrates, when kinetic limitations of mass transfer is taken into account, the largest concentrations of nitric acid are in the particles which have a fairly high number density. For example, in the simulation case $\mathrm{B}$, the number concentration of the particles that form the larger mode is approximately $1.3 \mathrm{~cm}^{-3}$

Figure 10 shows the partitioning of nitric acid between the large droplets which have exceeded their open system Köhler curve maxima and small "interstitial" droplets for case A. In Fig. 10, the blue line shows the liquid phase concentration in the large droplets, and the red line the concentration in the small droplets.

When the concentration of nitric acid in large droplets is compared to the total liquid phase nitric acid concentration (black dashed line), we can see that almost all of the nitric 
acid is in the largest droplets. Also, the gas phase concentration (blue dashed line) shows that nitric acid is efficiently depleted during the simulation.

\section{Conclusions}

We have extended the Köhler theory for aqueous solution droplets in the presence of a soluble trace gas to describe the equilibrium behavior of polydisperse droplet populations as a function of RH. Our equilibrium calculations for a closed system containing nitric acid show that as relative humidity grows enough, the uptake of $\mathrm{HNO}_{3}$ leads to splitting of the size distribution in such a way that the droplets in the largest size bin of the population start growing steeply as a function of RH, whereas the smaller droplets lose some of their water and nitric acid. The splitting can be understood in terms of open system Köhler curves which are calculated assuming a constant amount of gas-phase $\mathrm{HNO}_{3}$. Considering the closed system (with respect to nitric acid), at a given RH some concentration of nitric acid remains in the gas-phase, and the closed system Köhler curve crosses the open system Köhler curve calculated for that specific gas-phase $\mathrm{HNO}_{3}$ concentration. Enhanced uptake of $\mathrm{HNO}_{3}$ results when the crossing point of the closed and open system Köhler curves approaches and passes the maxima on the open system curves.

When mass transfer limitations of the soluble trace gas are accounted for, it can be seen the splitting of the size distribution shifts toward smaller droplet sizes. The gap appears at smaller and smaller sizes when the relative humidity is increased faster. As an application of the theory, we showed that stratospheric supercooled ternary solution droplet populations may experience the splitting and become bimodal. The calculated size distributions match the measured size distributions for which the larger particle mode consisted of NAT (Voigt et al., 2000). Our calculations show that the liquid phase molar ratio of $\mathrm{H}_{2} \mathrm{O}: \mathrm{HNO}_{3}$ may decrease close to 3 in the larger droplet mode, indicating that under suitable conditions, NAT size distributions similar to those measured by Voigt et al. (2000) could form in the stratosphere due to splitting of the STS distribution, followed by freezing of the larger mode to NAT.

Acknowledgements. This work was supported by the Academy of Finland.

\section{References}

Carslaw, K., Peter, T., and Clegg, S.: Modeling the composition of liquid stratospheric aerosols, Rev. Geophys., 35, 125-154, 1997.

Clegg, S. L., Brimblecombe, P., and Wexler, A. S.: Thermodynamical model of the system $\mathrm{H}^{+}-\mathrm{NH}_{4}^{+}-\mathrm{SO}_{4}^{2-}-\mathrm{NO}_{3}^{-}-\mathrm{H}_{2} \mathrm{O}$ at tropospheric temperatures, J. Phys. Chem. A, 102, 2137-2154, 1998.
Jacobson, M. Z.: Studying the effects of calcium and magnesium on size-distributed nitrate and ammonium with EQUISOLV II, Atmos. Environ., 33, 3635-3649, 1999.

Kärcher, B. and Solomon, S.: On the composition and optical extinction of particles in the tropopause region, J. Geophys. Res., 104, 27 441-27 459, 1999.

Köhler, H.: The nucleus in the growth of hygroscopic droplets, Trans. Faraday Soc., 32, 1152-1161, 1936.

Kokkola, H., Romakkaniemi, S., and Laaksonen, A.: On the formation of radiation fogs under heavily polluted conditions, Atmos. Chem. Phys., 3, 581-589, 2003a.

Kokkola, H., Romakkaniemi, S., and Laaksonen, A.: A onedimensional cloud model including trace gas condensation and sulfate chemistry, (submitted to Boreal Env. Res.), 2003b.

Konopka, P. and Vogelsberger, W.: Köhler equation for finite systems - a simple estimation of possible condensation mechanisms in aircraft contrails, J. Geophys. Res., 102, 16 057-16 064, 1997.

Kulmala, M., Laaksonen, A., Korhonen, P., Vesala, T., Ahonen, T., and Barrett, J. C.: The effect of atmospheric nitric acid vapor on cloud condensation nucleus activation, J. Geophys. Res., 98, 22 949-22 958, 1993.

Kulmala, M., Laaksonen, A., Charlson, R. J., and Korhonen, P.: Clouds without supersaturation, Nature, 388, 336-337, 1997.

Laaksonen, A., Korhonen, P., Kulmala, M., and Charlson, R. J.: Modification of the Köhler equation to include soluble trace gases and slightly soluble substances., J. Atmos. Sci., 55, 853862, 1998.

Martin, E., George, C., and Mirabel, P.: Densities and surface tensions of $\mathrm{H}_{2} \mathrm{SO}_{4} / \mathrm{HNO}_{3} / \mathrm{H}_{2} \mathrm{O}$ solutions., Geophys. Res. Lett., 27, 197-200, 2000.

Rizi, V. and Visconti, G.: Physical analogs and performance of a box model for composition and growth of $\mathrm{H}_{2} \mathrm{SO}_{4} / \mathrm{H}_{2} \mathrm{O}$ and $\mathrm{HNO}_{3} / \mathrm{H}_{2} \mathrm{SO}_{4} / \mathrm{H}_{2} \mathrm{O}$ aerosol in the stratosphere, J. Aerosol Sci., 30, 1095-1113, 1999.

Seinfeld, J. H. and Pandis, S. N.: Atmospheric Chemistry and Physics: From air pollution to climate change, Cloud Physics, 777-840, John Wiley \& Sons Inc., 1998.

Shulman, M. L., Jacobson, M. C., Charlson, R. J., Synovec, R. E., and Young, T. E.: Dissolution behavior and surface tension effects of organic compounds in nucleating cloud droplets, Geophys. Res. Lett., 23, 277, 1996.

Tabazadeh, A., Turco, R. P., and Jacobson, M. Z.: A model for studying the composition and chemical effects of stratospheric aerosols, J. Geophys. Res., 99, 12 897-12 914, 1994.

Tabazadeh, A., Martin, S. T., and Lin, J.-S.: The effect of particle size and nitric acid uptake on an homogenous freezing of aqueous sulfuric acid particles, Geophys. Res. Lett., 27, 1111-1114, 2000.

Voigt, C., Schreiner, J., Kohlmann, A., Zink, P., Mauersberger, K., Larsen, N., Deshler, T., Kröger, C., Rosen, J., Adriani, A., Cairo, F., Di Donfransesco, G., Viterbini, M., Ovarlez, J., Ovarlez, H., David, C., and Dörnbrack, A.: Nitric Acid Trihydrate (NAT) in Polar Stratospheric Clouds, Science, 290, 1756-1758, 2000. 